\title{
Unified studies of structure and reactions in light unstable nuclei
}

\author{
Makoto Ito ${ }^{1, a}$ \\ ${ }^{1}$ Department of Pure and Applied Physics, Kansai University, 3-3-35 Yamate-cho, Suita, 564-8680, Japan
}

\begin{abstract}
The generalized two-center cluster model (GTCM), which can treat covalent, ionic and atomic configurations in general systems with two inert cores plus valence nucleons, is formulated in the basis of the microscopic cluster model. In this model, the covalent configurations constructed by the molecular orbital (MO) method and the atomic (or ionic) configuration obtained by the valence bonding (VB) method can be described in a consistent manner. GTCM is applied to the light neutron-rich system, ${ }^{10,12} \mathrm{Be}=\alpha+\alpha+\mathrm{XN}(\mathrm{X}=2,4)$, and the unified studies of the structural changes and the reaction problem are performed. In the structure study, the calculated energy levels are characterized in terms of the chemical bonding like structures, such as the covalent MO or ionic VB structures. The chemical bonding structures changes from level to level within a small energy interval. In the unbound region, the structure problem with the total system of $\alpha+\alpha+\mathrm{XN}$ and the reaction problem, induced by the collision of an asymptotic VB state of $\alpha{ }^{6,8} \mathrm{He}$, are combined by GTCM. The properties of unbound resonant states are discussed in a close connection to the reaction mechanism, and some enhancement factors originated from the properties of the intrinsic states are predicted in the reaction observables. The unified calculation of the structures and the reactions is applied to the Coulomb shift problem in the mirror system, such the ${ }^{10} \mathrm{Be}$ and ${ }^{10} \mathrm{C}$ nuclei. The Coulomb displacement energy of the mirror systems are discussed.
\end{abstract}

\section{Introduction}

In light neutron-excess systems, many kinds of molecular structures are discussed from the viewpoint of the clustering phenomena. In particular, much attention has been concentrated on Be isotopes from theoretical and experimental approaches. The Be isotopes can be considered as typical examples of two-center superdeformed systems which build on an $\alpha+\alpha$ rotor of ${ }^{8} \mathrm{Be}$. Various cluster structures are generated by the coupling the two $\alpha$ degrees of freedom and the single particle motion of the excess neutrons. The coupled structures of the two $\alpha$ cores and the valence neutrons are analogous to the so-called chemical-bonding structure in molecular systems.

The low-lying states can be nicely understood by the molecular orbital (MO) picture, in which the excess neutrons perform the single-particle motion in the covalent orbitals, such as $\pi^{-}$or $\sigma^{+}$, around two $\alpha$ cores [1-3]. The typical application of the MO model is demonstrated in ${ }^{10} \mathrm{Be}=\alpha+\alpha+N+N$ [2]. In the MO model, the covalent orbitals are constructed by the technique of the linear combination of the atomic orbital (LCAO) [1,2]. Since the nuclear interaction of two $\alpha$ cores is weak, the $\alpha-\alpha$ relative motion strongly couples to the configuration of the LCAO covalent orbitals. For example, the

ae-mail: itomk@kansai-u.ac.jp 
$\sigma^{+}$orbit, which has the enlarged distribution along the $\alpha-\alpha$ axis, enhances the $\alpha-\alpha$ clustering. The $\sigma^{+}$orbital, which contains a large S-wave component, plays an important role for the formation of the low-lying intruder $0^{+}$state [3].

On the contrary, in the highly-excited region of $E_{x} \geq 10 \mathrm{MeV}$, recent experiments revealed the existence of the interesting resonant states, which dominantly decay into the ${ }^{4,6,8} \mathrm{He}$ fragments [4-6]. The data has been accumulated especially in ${ }^{12} \mathrm{Be}$, and these resonances are interpreted in terms of the ionic or atomic configurations of ${ }^{x} \mathrm{He}+{ }^{y} \mathrm{He}$, in which valence neutrons are trapped in one of an $\alpha$ cores [7, 8]. The localization of the valence neutrons around a core is very different from the MO picture. In the quantum chemistry, such the localized orbitals are usually treated by the so-called the valence bonding (VB) method. Therefore, the observation of the highly-excited resonances means that the structural changes occurs from the low-lying MO structures to the highly-excited VB structures.

The structural change from MO to VB is an interesting research subject. In order to handle these two configurations in a consistent manner, the traditional MO model based on the LCAO technique should be improved to cover the VB configuration. Recently, we have formulated the generalized twocenter cluster model (GTCM), which is available to treat both of the MO and VB configurations [7,8]. The GTCM can also describe not only the structure change of MO and VB but also the reaction process induced by the collision of the VB configuration in a unified manner. We have applied GTCM to the ${ }^{10,12} \mathrm{Be}$ nuclei, and the unified studies of the structure change and the respective reaction problem, such as the slow scattering of $\alpha+{ }^{6,8} \mathrm{He}$, have been performed [7, 8].

The studies on the mirror system of ${ }^{10,12} \mathrm{Be}$ is the naive extension of GTCM, and the analysis of the Coulomb shift is another interesting research subject. In the previous studies, for example, a characteristic Coulomb effect is known as the so-called Thomas-Ehrman shift (TES) in ${ }^{17} \mathrm{O}$ and ${ }^{17} \mathrm{~F}$ [9]. In the ${ }^{16} \mathrm{O}$ core plus one nucleon model, the Coulomb shift in ${ }^{17} \mathrm{~F}={ }^{16} \mathrm{O}+P$ is exceptionally suppressed for the S-wave orbit. Such the suppression of the Coulomb repulsion is expected in the $\alpha$ cluster structure because the development of the cluster structure usually involves the large mixture of the S-wave component. In the covalent MO picture, for example, the $\sigma^{+}$orbit with a large mixture of the $\mathrm{S}$-wave component enhance the $\alpha-\alpha$ clustering, while two nuclei dominantly perform the S-wave relative motion in the ionic VB states. Therefore, there is a possibility that the Coulomb suppression strongly reflects the development of the $\alpha$ cluster structure in the MO or VB configuration.

In this article, we demonstrate the unified studies of the structure changes in ${ }^{10,12} \mathrm{Be}$ and the reactions induced by the slow $\alpha+{ }^{6,8} \mathrm{He}$ scattering. Moreover, we extend GTCM to the mirror systems of ${ }^{10} \mathrm{Be}(=\alpha+\alpha+2 N)$ and ${ }^{10} \mathrm{C}(=\alpha+\alpha+2 P)$, and the the Coulomb shift of these mirror systems is discussed in connection to the cluster structures.

\section{Theoretical framework}

\subsection{Generalized Two-center Cluster Model}

The framework of the generalized two-center cluster model (GTCM) corresponds to the extension of the microscopic cluster model, which is based on the generator coordinate method (GCM) [10]. The detailed explanation of GTCM has already been published in Refs. [7, 8], and we briefly explain the formulation of GTCM by showing the example of two $\alpha$ plus two valence neutrons, such as ${ }^{10} \mathrm{Be}=\alpha$ $+\alpha+2 N$ and ${ }^{10} \mathrm{C}=\alpha+\alpha+2 P$. In this model, the basis function is given by

$$
\Phi_{\mathbf{m}}^{J^{\pi} K}(S)=\hat{P}_{K}^{J^{\pi}} \mathcal{A}\left\{\psi_{L}(\alpha) \psi_{R}(\alpha) \prod_{j=1}^{2} \varphi_{j}\left(m_{j}\right)\right\}_{S} .
$$


The $\alpha$-clusters at the left $(L)$ and right $(R)$ side center are shown by $\psi_{i}(\alpha)(i=L, R)$, and they are placed with a relative distance parameter of $S$, which is the generator coordinate. The internal structure of the $\alpha$ cluster is expressed by the $(0 s)^{4}$ configurations in the harmonic oscillator (HO) potential. The width parameter of the HO potential is set to $b=1.46 \mathrm{fm}$. The single-particle wave function for the valence neutrons localized around the $\alpha$ cluster is given by an atomic orbital (AO) $\varphi\left(m_{j}\right)$, which is labeled by the AO indices of $m_{j}=\left(p_{k}, i, \tau\right)$. Here, $p_{k}(k=x, y, z)$ means the $0 p$-orbitals around $i$-th core $(i$ $=L$ or $R$ ) with the spin $\tau(=\uparrow$ or $\downarrow)$. In Eq. (1), m represents a set of AOs for the valence neutrons, $\mathbf{m}=\left(m_{1}, m_{2}\right)$. The intrinsic basis functions with the full anti-symmetrization $\mathcal{A}$ are projected to the eigenstate of the total spin $J$, its intrinsic angular projection $K$, and the total parity $\pi$ by the projection operator $\hat{P}_{K}^{J^{\pi}}$.

The total wave function is finally given by taking the superposition over $S, \mathbf{m}$ and $K$ as

$$
\hat{\Psi}_{v}^{J^{\pi}}=\int d S \sum_{\mathbf{m}, K} C_{\mathbf{m} K}^{J^{\pi} v}(S) \Phi_{\mathbf{m}}^{J^{\pi} K}(S)
$$

In the present calculation, we consider all the possible $0 p$ configurations for the AO basis with $\mathbf{m}$, which leads to the complete formation of the covalent, ionic, and atomic configuration around two $\alpha$ cores. The coefficients $C_{\mathbf{m}}^{J^{\pi} v}(S)$ in Eq. (2) for the $v$-th eigenstate are determined by solving a coupled channel GCM (Generator Coordinate Method) equation [10]

$$
\left\langle\Phi_{\mathbf{m}}^{J^{\pi}}(S)\left|\left(\hat{H}-E_{v}^{J^{\pi}}\right)\right| \Psi_{v}^{J^{\pi}}\right\rangle=0
$$

The bound states below the $\alpha$ decay threshold are calculated by a diagonalization procedure, while the scattering boundary condition is explicitly imposed above the threshold, which will be explained in the later section. The solution of GCM equation can automatically reproduce the MO or VB structure, the formation of which depends on the situation. For example. the MO configurations are generated at the ground and low-lying bound states, while the VB configuration predominant in the continuum region above the $\alpha$ threshold.

In the present studies, the intrinsic configuration is restricted to the axial symmetric case $(K=$ 0 ) because we mainly consider the structure change in the $J^{\pi}=K^{\pi}=0^{+}$states. The restriction to the axial symmetric configurations can also be justified for the low-energy reaction phenomena in the finite spin states $\left(J^{\pi} \neq 0^{+}\right)$. The validity of the axial symmetric configuration is deeply discussed in the studies of the sub-barrier fusion reaction based on the time-dependent wave packet approach [11].

\subsection{Hamiltonian and effective nucleon-nucleon interaction}

The total Hamiltonian $\hat{H}$ in the present calculation is composed of the nucleon degrees of freedom and its explicit form is

$$
\hat{H}=\sum_{i}^{12} \hat{t}_{i}-\hat{T}_{c . m .}+\sum_{i<j}^{12} \hat{v}_{i j}^{(C)}+\sum_{i<j}^{12} \hat{v}_{i j}^{(L S)}+\sum_{i<j}^{12} \hat{v}_{i j}^{(\text {Coul })},
$$

where $i$ and $j$ denote a number of nucleons. $\hat{t}$ represents a single nucleon's kinetic energy, while $\hat{v}_{i j}^{(C)}$ and $\hat{v}_{i j}^{(L S)}$ are the two body nucleon-nucleon (NN) interactions for the central and spin-orbit parts, respectively. $\hat{v}_{i j}^{(\text {Coul })}$ represents the Coulomb interaction, which includes the projection operator to the proton state. The kinetic energy of the center of mass (c.m.) motion, shown by $\hat{T}_{c . m .}$, is subtracted from the summation of the nucleon's kinetic energy. In the present treatment, the c.m. motion of the total system can be exactly eliminated [10]. 
As for the NN interaction, we use the Volkov No.2 [12] and the G3RS [13] for the central $\hat{v}_{i j}^{(C)}$ and spin-orbit $\hat{v}_{i j}^{(l S)}$ parts, respectively. The functional form of the Volkov No.2 interaction is

$$
\hat{v}_{i j}^{(C)}=v^{(C)}\left(r_{i j}\right)\left(W-M P_{\sigma} P_{\tau}+B P_{\sigma}-H P_{\tau}\right)
$$

Here $v^{(C)}(r)$ is given by the superposition of Gaussian, while $W, M P_{\sigma} P_{\tau}, B P_{\sigma}$ and $H P_{\tau}$ denote the Wigner, Majorana, Bartlett and Heisenberg exchanges. The G3RS spin-orbit interaction has the functional form of

$$
\hat{v}_{i j}^{(L S)}=v^{(L S)}\left(r_{i j}\right) P\left({ }^{3} O\right) \mathbf{L}_{i j} \cdot \mathbf{S}_{i j},
$$

where $P\left({ }^{3} \mathrm{O}\right)$ represents the projection operator on the triplet-odd state for the two nucleon channel. $\mathbf{L}_{i j}=\mathbf{r}_{i j} \times \mathbf{p}_{i j}$ and $\mathbf{S}_{i j}=\mathbf{s}_{i}+\mathbf{s}_{j}$ are the relative and total nucleons' spins for interacting $i$-th and $j$-th nucleons, respectively. The form factor of $v^{(L S)}(r)$ is also constructed by the superposition of Gaussian.

A combination of Volkov. No. 2 and G3RS has already been applied to the previous MO models $[2,3]$. In the MO model, these interactions are successful in reproducing various properties observed in the low-lying states of ${ }^{10} \mathrm{Be}$, especially the formation of the intruder $0_{2}^{+}$state [3]. The details of the interaction parameters adopted in the present GTCM calculation are shown in Ref. [7]. These parameters are obtained by modifying the parameters used in the MO model [2,3], and they can nicely describe the properties of the low-lying states in ${ }^{10,12} \mathrm{Be}[7,8]$. Furthermore, the adopted parameters can also reproduce the threshold energies for the various ${ }^{x} \mathrm{He}+{ }^{y} \mathrm{He}$ channels, as shown in Ref. [7]. In ${ }^{12} \mathrm{Be}$, the energy difference of $\alpha+{ }^{8} \mathrm{He}_{\text {g.s. }}$ and ${ }^{6} \mathrm{He}_{\text {g.s. }}+{ }^{6} \mathrm{He}_{\text {g.s. }}$ in the calculation is a little larger than that in the experimental observation (about $1 \mathrm{MeV}$ ), but the relative differences among the thresholds are reasonably reproduced by the present $\mathrm{NN}$ interaction.

Since the threshold energy corresponds to the order of magnitude of the interaction strength to dissociate a compound system into a pair of He clusters, the reproduction of the threshold energy is crucial in discussing the appearance and disappearance of the cluster degrees of freedom. This means that the correct treatment of the threshold is indispensable for the unified studies of the structures and reactions, where dynamical changes of the cluster structure prominently occurs. Therefore, the NN interactions tuned in the present study, which reproduce the thresholds for various He decays as well as the low-lying states, can be considered quite reliable for the unified calculation of the structure and the reactions.

\subsection{Scattering boundary condition}

The superposition of the basis function in Eq. (1) over the possible $\mathbf{m}$ can reproduce the binary ${ }^{x} \mathrm{He}+$ ${ }^{y} \mathrm{He}$ dimer states in the asymptotic region of the distance parameter $S$ [7]. This binary states are the same as the asymptotic channels in the nuclear reaction problem. Thus, we can naturally impose the scattering boundary condition on the total wave function in Eq. (2), and the eigenvalue equation of Eq. (3) can be solved under the scattering boundary condition. There are two methods in solving the scattering problem: Kohn-Hulthén-Kato (KHK) method [8] and the closed state method [7]. These two methods are completely equivalent if the converged results are obtained within a given model space. Here we mainly explain the closed state method because this method is very convenient in the analysis of the formation and decays of the resonant levels excited in a reaction process. In the closed state method, a coupling of the closed compound states under the bound state approximation and the open channels with the scattering boundary is directly solved.

Let us explain the basic idea of the closed state method by showing an example of the ${ }^{12} \mathrm{Be}$ nuculeus. In this system, we consider two reactions induced by the scattering of ${ }^{8} \mathrm{He}_{\text {g.s. }}$ by an $\alpha$ 
target: the elastic scattering of $\alpha+{ }^{8} \mathrm{He}_{\text {g.s. }} \rightarrow \alpha+{ }^{8} \mathrm{He}_{\text {g.s. }}$ and two neutron transfer reaction of $\alpha+{ }^{8} \mathrm{He}_{\text {g.s. }}$. $\rightarrow{ }^{6} \mathrm{He}_{\text {g.s. }}+{ }^{6} \mathrm{He}_{\text {g.s. }}$. In a simple transition between these two open channels, the scattering (S) matrix reveals just a smooth energy dependence as will be shown in the later section. Thus, it is insufficient to describe resonance phenomena, in which there is a complicated energy dependence in S matrix. In order to handle resonance phenomena, we introduce the intermediate quasi-bound states, which have the more complicated structures than that of the open channels. The quasi-bound states are obtained by solving the eigenvalue problem in Eq. (3) in a finite-basis expansion method, in which the $\alpha-\alpha$ distance parameter $S$ is limited inside of the nuclear interaction range. This quasi-bound state is called the closed states because they are confined inside of the interaction region and never decay into asymptotic region. Reaction processes can be described in terms of the coupling of the open channels and the closed compound states: An incident wave in $\alpha+{ }^{8} \mathrm{He}_{\text {g.s. }}$ can couple to the closed states as well as final channels, and the incident wave is scattered into a final channel through the transitions among the closed states.

According to this simple image, the total wave function, which takes into account a coupling of open channels and closed states, can be constructed by a linear combination of these two parts like

$$
\Psi^{J^{\pi}(+)}=\sum_{\beta L} \varphi_{\beta L}^{J^{\pi}(+)}\left(\mathbf{R}_{\beta}\right)+\sum_{v} b_{v} \hat{\Psi}_{v}^{J^{\pi}}
$$

where the first term $\varphi_{\beta L}^{J^{\pi}(+)}\left(\mathbf{R}_{\beta}\right)$ stands for the open channels labeled by a channel subscript $\beta L$. Here $\beta$ means a set of the quantum number of a combination of the scattering particles (excitation energy, internal spin, and so on). $\varphi_{\beta L}^{J^{\pi}(+)}\left(\mathbf{R}_{\beta}\right)$ is given by the superposition of the channel basis, such as

$$
\varphi_{\beta L}^{J^{\pi}(+)}\left(\mathbf{R}_{\beta}\right)=\sum_{S} B_{\beta L}(S) \chi_{\beta L}^{J^{\pi}(+)}\left(\mathbf{R}_{\beta}, S\right) .
$$

In Eq. (8), $\chi_{\beta L}^{J^{\pi}(+)}\left(\mathbf{R}_{\beta}, S\right)$ shows the channel basis [7, 8], which is constructed from a specific linear combination of the AO basis in Eq. (1), while $B_{\beta L}(S)$ means the amplitude for the respective channel basis. The superscript $(+)$ of $\chi_{\beta L}^{J^{\pi}(+)}$ means that the scattering boundary condition for the incident and final open channels is explicitly imposed, according to the prescription of the KHK method [8].

The second term in Eq. (7) represents the closed compound states confined within the interaction region. The closed states are constructed by a linear combination of the solutions $\hat{\Psi}_{v}^{J^{\pi}}$ for Eq. (2) with an amplitude of $b_{v} . \hat{\Psi}_{v}^{J^{\pi}}$ is calculated by the standard diagonalization procedure, in which a range of the distance parameter $S$ is restricted inside of the interaction region.

We solve the Schrödinger equation $(H-E) \Psi^{J^{\pi}(+)}=0$ with the wave function assumed in Eq. (7). The equation can be reduced by eliminating the coefficients of $b_{v}$ in Eq. (7). When we substitute the total wave function in Eq. (7) into $(H-E) \Psi^{J^{\pi}(+)}=0$, the mixing coefficient $b_{v}$ for the closed state $\Psi_{v}^{J^{\pi}}$ can be written in the form of

$$
b_{v}=\frac{1}{E-E_{v}}<\hat{\Psi}_{v}^{J^{\pi}}|(H-E)| \sum_{\beta L} \varphi_{\beta L}^{J^{\pi}(+)}>.
$$

Here, $E_{v}$ is an energy eigenvalue of the intrinsic states, which satisfy the eigenvalue equation of Eq. (3) with the abbreviation of $E_{v}^{J^{\pi}} \rightarrow E_{v}$. Using Eq. (9), we can eliminate $b_{v}$ from the original Schrödinger equation and derive the effective equation for the open channels as

$$
(H-\mathcal{V}(E)-E) \sum_{\beta L} \varphi_{\beta L}^{J^{\pi}(+)}=0
$$


with the energy-dependent non-local interaction of

$$
\mathcal{V}(E)=\sum_{v}(H-E)\left|\Psi_{v}^{J^{\pi}}>\frac{1}{E-E_{v}}<\Psi_{v}^{J^{\pi}}\right|(H-E)
$$

This non-local interaction corresponds to the so-called dynamic polarization potential, which represents the coupling of the open channels and the intrinsic states, which have the more complicated structure than the incident and exit channels [14]. Equation (10) for the open channels can be solved by the standard technique of the KHK method [8], and the S matrix elements for the transition from the incident and exit channels are obtained.

\section{Results}

\subsection{Chemical bonding structure in ${ }^{10,12} \mathrm{Be}$}

The energy levels from the bound region to the unbound region are shown in the left $\left({ }^{10} \mathrm{Be}\right)$ and right $\left({ }^{12} \mathrm{Be}\right)$ panels of Fig. $1\left(J^{\pi}=0^{+}\right)$. In this figure, the ordinates represents the total energy with respect to the $\alpha$ threshold, and the energy levels become the bound and resonant states below and above the $\alpha$ threshold energy, respectively. The energy levels are shown up to the excitation energy of $E_{x} \leq 15$ $\mathrm{MeV}$. In this calculation, bound states are obtained by the diagonalization procedure, while resonant states are identified from the energy dependence of the scattering matrix in the complex plane.

In this figure, the total reaction probability, which is defined by $1-\mathfrak{R} S_{e l}$ with the elastic $\mathrm{S}$ matrix of $S_{e l}$ is also shown. The $\mathrm{S}$ matrix is calculated from the closed state method, explained at the previous section. In the reaction probability of ${ }^{10} \mathrm{Be}$, broad structure can be seen, while, in ${ }^{12} \mathrm{Be}$, there is complicated behavior in the reaction probability, which implies the existence of many resonant states with narrow width. At the resonance energy position, the various chemical bonding-like structures are generated, as shown by the schematic images attached on these two panels.

The obtained $0^{+}$states are classified into three categories according to analysis of the wave function as follows:

(i) Compact MO states: In ${ }^{10} \mathrm{Be}$, the MO state of $\left(\pi_{3 / 2}^{-}\right)^{2}$ and $\left(\pi_{1 / 2}^{-}\right)^{2}$, which form the parallel and anti-parallel spin-orbit pair, are generated at the ground $0_{1}^{+}$and the highest $0_{4}^{+}$state, respectively. In ${ }^{12} \mathrm{Be}$, the four neutrons are in MO motion around the two $\alpha$ cores in two bound states $\left(\left(\pi_{3 / 2}^{-}\right)^{2}\left(\sigma_{1 / 2}^{+}\right)^{2}\right.$ in $0_{1}^{+}$and $\left(\pi_{3 / 2}^{-}\right)^{2}\left(\pi_{1 / 2}^{-}\right)^{2}$ in $\left.0_{2}^{+}\right)$, appearing below the $\alpha+{ }^{8} \mathrm{He}_{\text {g.s. }}$ threshold. The $\pi_{3 / 2}^{-}$and $\pi_{1 / 2}^{-}$orbits, corresponding to the $0 p_{3 / 2}$ and $0 p_{1 / 2}$ orbits in the shell model limit, respectively, gain the binding energy in the case of the compact $\alpha-\alpha$ distance. Therefore, the MO states containing the $\pi_{3 / 2}^{-}$and $\pi_{1 / 2}^{-}$orbits have the spatially compact structures.

(ii) Extended VB states with ${ }^{x} \mathrm{He}+{ }^{y} \mathrm{He}$ : Above the $\alpha$ decay threshold, ionic He-dimer states, which have the VB structure of excess neutrons, are generated in both ${ }^{10,12} \mathrm{Be}$. In ${ }^{10} \mathrm{Be}$, for instance, the $\alpha+{ }^{6} \mathrm{He}\left(2_{1}^{+}\right)$structure appears in the $0_{3}^{+}$state, which is a resonant state around $E \sim 4 \mathrm{MeV}$. The box in the left panel corresponds to the $0^{+}$state having the dominant $\alpha+{ }^{6} \mathrm{He}_{\text {g.s. }}$ structure, but this $0^{+}$state is not prominent resonance but continuum like state. The hight of the box shows the width of the $\alpha+{ }^{6} \mathrm{He}_{\text {g.s. }}$. continuum state. As shown in the reaction probability, this continuum state is observed as a broad peak in the $\alpha+{ }^{6} \mathrm{He}_{\text {g.s. }}$ scattering. The resonant states, $0_{3}^{+}, 0_{4}^{+}$, and $0_{6}^{+}$in ${ }^{12} \mathrm{Be}$ have the He-dimer configuration of the corresponding threshold, $\alpha+{ }^{8} \mathrm{He}_{\text {g.s }},{ }^{6} \mathrm{He}_{\text {g.s. }}+{ }^{6} \mathrm{He}_{\text {g.s. }}$, and ${ }^{5} \mathrm{He}_{\text {g.s. }}$. $+{ }^{7} \mathrm{He}_{\text {g.s. }}$, respectively. In these He-dimer resonances, the relative wave function between two $\mathrm{He}$ nuclei is largely extended and hence, these resonant states can be interpreted in terms of the molecular resonances, composed of two He fragments. 


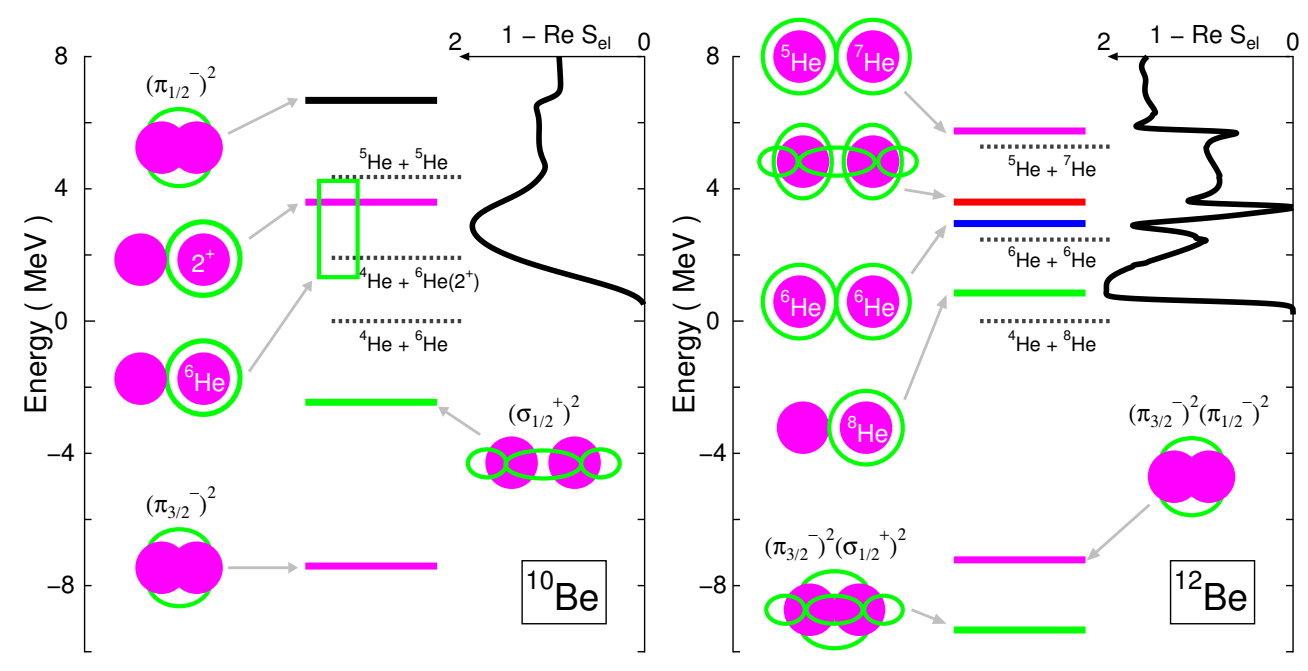

Figure 1. (Color online) Left panel: The energy spectra of ${ }^{10} \mathrm{Be}\left(J^{\pi}=0^{+}\right)$. The solid curve on the right sides shows the total reaction probability of the $\alpha+{ }^{6} \mathrm{He}_{\text {g.s. }}$. scattering with a respective scale at the top most axis. The dotted lines represent the threshold energy of the open channels considered in the calculation. The box represents the broad continuum state. Right panel: The same as left panel except for ${ }^{12} \mathrm{Be}$. These figures are taken from Ref. [7]

(iii) Covalent SD state: The $0_{2}^{+}$state of ${ }^{10} \mathrm{Be}$ has the $\left(\sigma_{1 / 2}^{+}\right)^{2}$ configuration. The structure of $0_{5}^{+}$ state in ${ }^{12} \mathrm{Be}$ is based on ${ }^{10} \mathrm{Be}\left(0_{2}^{+}\right)$. The main component of this state is the product of $\left(\sigma_{1 / 2}^{+}\right)^{2} \otimes\left({ }^{5} \mathrm{He}\right.$ $\left.+{ }^{5} \mathrm{He}\right)$. Due to the $\left(\sigma_{1 / 2}^{+}\right)^{2}$ formation, these states have a large clustering $(S \sim 5 \mathrm{fm})$ in comparison with the touching distance of two $\alpha$ particles $(\sim 2.8 \mathrm{fm})$.

In both the systems, the compact MO states appear in the bound region below the $\alpha$ threshold, while the compact MOs and the extended VB states coexist in continuum region; specifically, the spatially compact and extended states coexist in the same energy region. This is in marked contrast to the naive expectation that the spatial extension of two $\alpha$ cores monotonically increase as a function of an excitation energy. In ${ }^{10} \mathrm{Be}$, we can observe a wide variety in the structure change, which are induced by the coupling of the two excess neutrons. In ${ }^{12} \mathrm{Be}$ with four excess neutrons, furthermore, more levels are generated in the same energy region of $E_{x} \leq 15 \mathrm{MeV}$, and hence the structure changes becomes much more prominent in comparison to ${ }^{10} \mathrm{Be}$.

Let us compare the level scheme in Fig. 1 with the recent experiments. In ${ }^{10} \mathrm{Be}$, shown in the left panel of Fig. 1, the theoretical energy of the intruder $0_{2}^{+}$state nicely reproduces the observed excitation energy. Furthermore, the broad $0^{+}$strength is identified by the experiment by Rogachev et al. [16], and the energy position of the observed strength corresponds to the calculated continuum $0^{+}$state just above the $\alpha+{ }^{6} \mathrm{He}_{\text {g.s. }}$ threshold. The calculation of ${ }^{12} \mathrm{Be}$ also reproduces the recent observations nicely. In the bound region, the excitation energy of the intruder $0_{2}^{+}$state in ${ }^{12} \mathrm{Be}$ is much lower than that in ${ }^{10} \mathrm{Be}$, and the energy of $\mathrm{O}_{2}^{+}$is almost same as the observed energy. In continuum region, theoretical calculation generates four levels with a close energy spacing, and such a degenerate feature of the energy levels are observed in the recent experiment of the inelastic scattering of ${ }^{12} \mathrm{Be}[5,6]$. In the inelastic scattering in Ref. [5], the observed $0^{+}$strength reveals the overlapping resonances with an energy distance of $\sim 1 \mathrm{MeV}$. Furthermore, there are at least two observed levels consistent with the 
calculated levels; the calculated $\mathrm{O}_{3}^{+}$and $\mathrm{O}_{5}^{+}$states has a dominant decay width into the $\alpha+{ }^{8} \mathrm{He}_{\text {g.s. }}$ and ${ }^{6} \mathrm{He}_{\text {g.s. }}+{ }^{6} \mathrm{He}_{\text {g.s. }}$ channels, respectively, and the two levels having similar decay schemes are observed around the same energy regions $[5,6]$. The decay scheme will be discussed in the later sections.

\subsection{Threshold energies and the resonant levels}

In the $N=Z$ nuclear system, the $\alpha$ cluster structures are known to appear according to the threshold rule, in which the cluster structures appear around the excitation energy close to the threshold energy dissociating into the corresponding cluster configuration [15]. It is interesting to focus on the relation of the continuum states in ${ }^{10,12} \mathrm{Be}$ and threshold energies from the viewpoint of the threshold rule. A comparison of the levels in continuum region with the respective He-decay thresholds is summarized in two panels of Fig. 2. In these panels, the four-body $\left({ }^{10} \mathrm{Be}=\alpha+\alpha+2 N\right)$ and six-body $\left({ }^{12} \mathrm{Be}=\alpha\right.$ $+\alpha+4 N)$ channels are also plotted although the degree of freedom for these many-body decay is not considered in the present calculation. Since, in ${ }^{10} \mathrm{Be}$, the calculated threshold difference, $\Delta E=$ $E_{t h}\left({ }^{5} \mathrm{He}_{\text {g.s. }}+{ }^{5} \mathrm{He}_{\text {g.s. }}\right)-E_{\text {th }}\left(\alpha+{ }^{6} \mathrm{He}\left(2_{1}^{+}\right)\right.$is larger by about $1.4 \mathrm{MeV}$ than the experimental value [7], the thresholds and the energy levels and thresholds above $E_{t h}\left(\alpha+{ }^{6} \mathrm{He}\left(2_{1}^{+}\right)\right)$are shifted by $1.4 \mathrm{MeV}$ to the lower energy side. Due to a similar reason, in ${ }^{12} \mathrm{Be}$, the thresholds and the energy levels above $E_{\text {th }}\left({ }^{6} \mathrm{He}_{\text {g.s. }}+{ }^{6} \mathrm{He}_{\text {g.s. }}\right)$ are shifted by $1.3 \mathrm{MeV}$.


Figure 2. (Color online) Left panel: Energy levels in the higher energy region in ${ }^{10} \mathrm{Be}$. The threshold for the binary He channels (calculation) and the $\alpha+\alpha+2 N$ (experiment) are shown by the dotted lines, while the energy levels are plotted by the solid lines. Right panel: The same as left panel but for ${ }^{12} \mathrm{Be}$. In ${ }^{10} \mathrm{Be}$, the threshold and the energy levels above the $\alpha+{ }^{6} \mathrm{He}\left(2_{1}^{+}\right)$threshold is shifted to the lower energy side by about $1.4 \mathrm{MeV}$, while, in ${ }^{12} \mathrm{Be}$, those above the ${ }^{6} \mathrm{He}_{\text {g.s. }}+{ }^{6} \mathrm{He}_{\text {g.s. }}$ threshold is shifted to the lower energy side by about $1.3 \mathrm{MeV}$. These figures are taken from Ref. [7]

In the left panel $\left({ }^{10} \mathrm{Be}\right)$, there is one to one correspondence between calculated levels and the threshold energies although the $0^{+}$state in a box is a broad continuum-like state. The discrete bound or resonant levels are distributed in an energy range of about $8 \mathrm{MeV}$, which corresponds to about 3 $\mathrm{MeV}$ energy interval on average. In marked contrast to the level scheme in ${ }^{10} \mathrm{Be}$, all the energy levels in ${ }^{12} \mathrm{Be}$ shown in the right panel are confined within the range of $\sim 4 \mathrm{MeV}$, and the energy interval between neighboring levels is just $\sim 1 \mathrm{MeV}$. Although the energy interval in ${ }^{12} \mathrm{Be}$ is much smaller 
than that in ${ }^{10} \mathrm{Be}$, we can still confirm the one to one correspondence between the energy levels and thresholds.

Because of the small spacings of the threshold energies in ${ }^{12} \mathrm{Be}$, the mixing of the He-dimer components is strong, although the dominant component can be identified in each of the energy levels. The dominant populations in the $0_{1}^{+} \sim 0_{6}^{+}$levels are investigated in Ref. [7], and they have been found to be about $50 \%$ on average. Thus, each level contains impurities besides a main component. Such a mixture of the impurities is important for reducing the decay width of the resonant levels, leading to the formation of the sharp resonance [7].

\subsection{Analysis of the low-energy $\alpha$ scattering}

\section{3. $1 \alpha+{ }^{8}$ He resonant scattering}

By solving exactly the effective equation for the open channels shown in Eq. (10), we calculate the $\mathrm{S}$ matrices for the elastic scattering and neutron transfer reactions, which are shown in Fig. 3. In this figure, $\mathrm{S}$ matrices $S_{f, i}$ for the transitions from the incident $\alpha+$ ${ }^{8} \mathrm{He}_{\text {g.s. }}$ channel $(i)$ to the final channels $(f)$, ${ }^{6} \mathrm{He}_{\text {g.s. }}+{ }^{6} \mathrm{He}_{\text {g.s. }}$ or ${ }^{5} \mathrm{He}_{\text {g.s. }}+{ }^{7} \mathrm{He}_{\text {g.s. }}$, are plotted as a function of the center-of-mass energy of the incident channel. In this figure, we can clearly understand the important role of the intrinsic states $\Psi_{v}^{J^{\pi}}$ in forming the sharp resonances.

The dotted curves represent the $\mathrm{S}$ matrices calculated only from the coupling of the open channels $\varphi_{\beta L}^{J^{\pi}(+)}$, which is included in the first term of Eq. (7). No resonant structures with sharp widths exist in the results of the open channels. In the full solutions shown by the solid curves, the sharp structures are generated by the coupling with the intrinsic states (double squares labeled by $0_{3}^{+} \sim 0_{6}^{+}$). Each peak is generated by a linear combination of all the intrinsic states, but the dominant contribution in the individual peak is a single intrinsic state, which is indicated by the arrows. Since the intrinsic states include the component of not only the open channels, but also of many kinds of closed channels, which have a higher excitation energy than the resonance energy, the coupling with the intrinsic states plays an important role in reducing the resonance width.

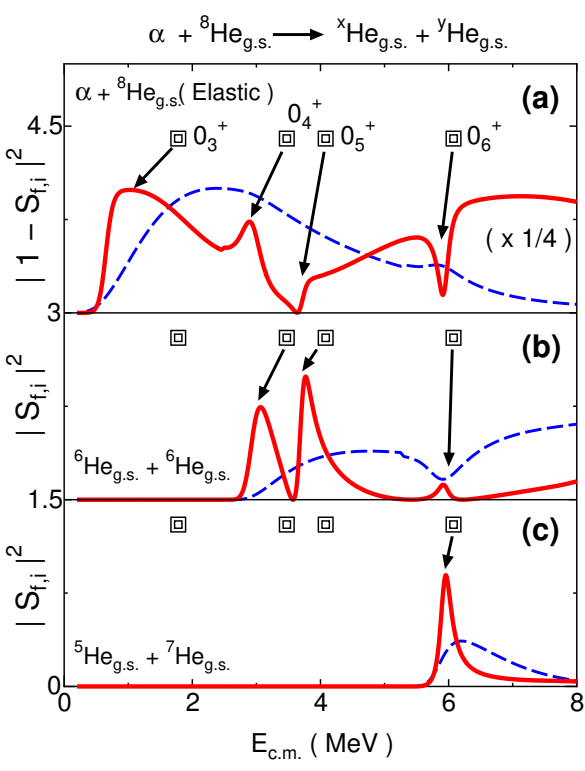

Figure 3. (Color online) $\mathrm{S}$ matrices for the central collision of $\alpha+{ }^{8} \mathrm{He}_{\text {g.s. }}\left(\mathrm{J}^{\pi}=0^{+}\right)$. Panel (a) shows the $\mathrm{S}$ matrix of the elastic scattering, while (b) and (c) show those for the final channels of ${ }^{6} \mathrm{He}_{\text {g.s. }}+{ }^{6} \mathrm{He}_{\text {g.s. }}$ and ${ }^{5} \mathrm{He}_{\text {g.s. }}+{ }^{7} \mathrm{He}_{\text {g.s. }}$, respectively. The dashed and solid curves represent the results calculated with the three open channels and the full solutions of Eq. (8), respectively. The double squares represent the energy levels obtained by the diagonalization of the total Hamiltonian. The $\mathrm{S}$ matrices of the elastic scattering are divided by a factor of four to compare with the magnitude of the non-elastic S matrices. This figure is taken from Refs. [7, 8].

It is naively expected that the coupled-channel calculation between the incident and exit channels is sufficient in describing the two-neutron transfer reaction, $\alpha+{ }^{8} \mathrm{He}_{\text {g.s }} \rightarrow{ }^{6} \mathrm{He}_{\text {g.s. }}+{ }^{6} \mathrm{He}_{\text {g.s. }}$. In the 
present calculation of the open channels, the ${ }^{5} \mathrm{He}_{\text {g.s. }}+{ }^{7} \mathrm{He}_{\text {g.s. }}$ channel, which is never considered in the naive two neutron transfer approach, is included. However, no sharp resonances appear even if the one-neutron transfer channel is coupled to the incident and final channels. The inclusion of the intrinsic states, which have a more complicated structure than those of the incident and exit channels, is essential in describing the formation of resonances with sharp widths.

The present calculation predicts the sharp resonances corresponding to the four levels, $0_{3}^{+} \sim 0_{6}^{+}$, shown in Fig. 2, in all the exit channels. The ratio of $\mathrm{S}$ matrices for different exit channels gives important information on the intrinsic structures of the compound system, ${ }^{12} \mathrm{Be}$. In particular, the resonant behavior around $E_{c . m .} \sim 4 \mathrm{MeV}(E \sim 1.3 \mathrm{MeV}$ with respect to the ${ }^{6} \mathrm{He}_{\text {g.s }}+{ }^{6} \mathrm{He}_{\text {g.s. }}$ threshold) gives an interesting result. At the resonance energy corresponding to $0_{5}^{+}$, the magnitude of the respective $S$ matrix element increases up to $\sim 1$. Since the unitary condition of $\sum_{f}\left|S_{f, i}\right|^{2}=1$ holds in the present calculation without any imaginary potentials, this enhancement means that the incident flux flows mainly into the ${ }^{6} \mathrm{He}_{\text {g.s. }}+{ }^{6} \mathrm{He}_{\text {g.s. }}$ channel. Around this enhancement region, $S_{e l}$ is quite small due to the unitary condition, and $\left|1-S_{e l}\right|^{2}$ becomes $\sim 1$. (Fig. 3(a)). This situation corresponds to the so-called shadow scattering in the elastic channel, in which the incident flux is strongly absorbed inside the interaction region and scattered as edge waves

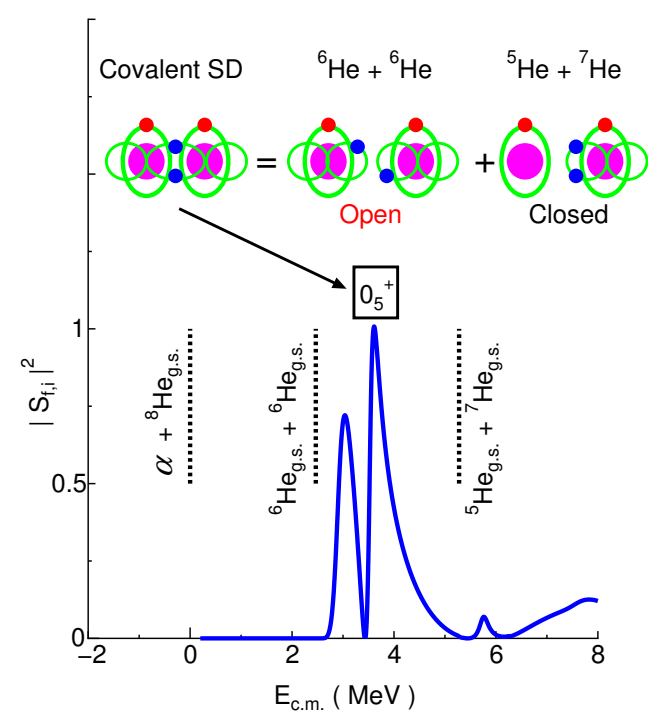

Figure 4. (Color) $\mathrm{S}$ matrix for the two neutron-transfer reactions, $\alpha+{ }^{8} \mathrm{He}_{\text {g.s. }} \rightarrow{ }^{6} \mathrm{He}_{\text {g.s. }}+{ }^{6} \mathrm{He}_{\text {g.s. }}\left(J^{\pi}=0^{+}\right)$. The dotted lines represent the threshold energy of the open channels considered in the calculation. In the upper part, the schematic picture of the covalent SD state is shown. This figure is taken from Refs. [7, 8] around the boundary of the strong absorber.

The result of $\left|S_{f, i}\right|^{2} \sim 1$ means that the $0_{5}^{+}$ state mainly decays into not $\alpha+{ }^{8} \mathrm{He}_{\text {g.s. }}$ but ${ }^{6} \mathrm{He}_{\text {g.s. }}+{ }^{6} \mathrm{He}_{\text {g.s. }}$. Such a characteristic decay scheme is due to the intrinsic structure of $0_{5}^{+}$, which has the covalent SD structure as explained in the previous section. The $\mathrm{S}$ matrix of two neutron transfer and the threshold energies of open channels are shown in Fig. 4. Since the $\sigma^{+}$orbital is constructed by $0 p_{z}(L)-0 p_{z}(R)$ where $0 p_{z}(C)$ represents the $0 p_{z^{-}}$ wave with the center $C$, the covalent SD is given by a linear combination of the $\left({ }^{6} \mathrm{He}+{ }^{6} \mathrm{He}\right)+\left({ }^{5} \mathrm{He}\right.$ $+{ }^{7} \mathrm{He}$ ) configurations, as shown in the schematic picture in Fig. 4. Here, ${ }^{6} \mathrm{He}+{ }^{6} \mathrm{He}$ and ${ }^{5} \mathrm{He}+{ }^{7} \mathrm{He}$ are constructed by a coherent mixing of the asymptotic channels of the respective configurations. A component of $\alpha+{ }^{8} \mathrm{He}_{\text {g.s. }}$, which is an open channel at the resonance energy, is suppressed in this configuration. Furthermore, the resonance energy is lower than the threshold of ${ }^{5} \mathrm{He}_{\text {g.s. }}+{ }^{7} \mathrm{He}_{\text {g.s. }}$, which is the lowest open channel in the ${ }^{5} \mathrm{He}+{ }^{7} \mathrm{He}$ configuration. Therefore, the decay channel of the covalent SD is mainly restricted to ${ }^{6} \mathrm{He}_{\text {g.s. }}+{ }^{6} \mathrm{He}_{\text {g.s. }}$. This is the reason why the $\mathrm{S}$ matrix corresponding to the covalent SD reaches the unitary limit of $\left|S_{f, i}\right|^{2} \sim 1$. Due to this limitation of the decay channel, the covalent SD becomes an isomeric state with a width of $\Gamma \sim 400 \mathrm{keV}$.

The enhancement of the decay into ${ }^{6} \mathrm{He}_{\text {g.s. }}+{ }^{6} \mathrm{He}_{\text {g.s. }}$ can be supported by a recent measurement of an inelastic $\alpha$ scattering on ${ }^{12} \mathrm{Be}$ [5]. In this study, the excitation energy spectrum of ${ }^{12} \mathrm{Be}$ was obtained from the invariant mass of the decay products, ${ }^{6} \mathrm{He}_{\text {g.s. }}+{ }^{6} \mathrm{He}_{\text {g.s. }}$ and $\alpha+{ }^{8} \mathrm{He}_{\text {g.s. }}$. The contributions 
of $0^{+}$were extracted by the multipole decomposition analysis (MDA) [5]. A sharp resonance was suggested in the ${ }^{6} \mathrm{He}_{\text {g.s. }}+{ }^{6} \mathrm{He}_{\text {g.s. }}$ decay channel about $1.2 \mathrm{MeV}$ above the decay threshold. In contrast, the corresponding resonance was not identified in the $\alpha+{ }^{8} \mathrm{He}_{\text {g.s. }}$ channel, suggesting a smaller partial decay width compared to the ${ }^{6} \mathrm{He}_{\text {g.s. }}+{ }^{6} \mathrm{He}_{\text {g.s. }}$ channel [5]. These observations are consistent with the predicted nature of the covalent isomeric state in terms of excitation energy and decay scheme. Furthermore, the low-energy ${ }^{8} \mathrm{He}$ scattering by an $\alpha$ target has been measured at GANIL [17]. Thus, the direct comparison of the theoretical calculation of the $\alpha$ scattering with the respective experiment will be possible in future studies.

\subsection{2 $\alpha+{ }^{6}$ He resonant scattering}

The $\mathrm{S}$ matrices in the $\alpha+{ }^{6} \mathrm{He}_{\text {g.s. }}$ reaction with $J^{\pi}=0^{+}$are calculated by applying the standard technique of the Kohn-Hulthén-Kato method [7, 8]. The calculated $\mathrm{S}$ matrices are shown in Fig. 5. In the panels of (a), (b) and (c), the $\mathrm{S}$ matrices going to the elastic $\alpha+$ ${ }^{6} \mathrm{He}_{\text {g.s. }}, \alpha+{ }^{6} \mathrm{He}\left(2+{ }_{1}^{+}\right),{ }^{5} \mathrm{He}_{\text {g.s. }}+{ }^{5} \mathrm{He}_{\text {g.s. }}(I=0)$ channels are plotted, respectively. We can confirm the broad resonant structures in the individual panels. There are two dents in the elastic channel (a) at $E_{c . m .} \sim 4 \mathrm{MeV}$ and 7 $\mathrm{MeV}$, while two peaks appear at the respective dent-energies in the non-elastic channels of (b) and (c). This anti-correlation of the $S$ matrices means that the incident flux from the elastic channel mainly absorbed in the specific exit channels, $\alpha+{ }^{6} \mathrm{He}\left(2_{1}^{+}\right)$and ${ }^{5} \mathrm{He}_{\text {g.s. }}$. $+{ }^{5} \mathrm{He}_{\text {g.s. }}(I=0)$, and resonances are generated in these exit channels. These broad structures are in marked contrast to the sharp structures appearing in the $\alpha+{ }^{8} \mathrm{He}_{\text {g.s. }}$ reaction shown in Fig. 3. Since the number of the excess neutrons in ${ }^{10} \mathrm{Be}(N=2)$ is smaller than that in ${ }^{12} \mathrm{Be}(N=4)$, the attraction from excess neutrons is not so strong in ${ }^{10} \mathrm{Be}$ in a comparison to ${ }^{12} \mathrm{Be}$. Due to this reduced attraction, the resonance width becomes much broader in ${ }^{10} \mathrm{Be}$.

We also apply the absorbing boundary

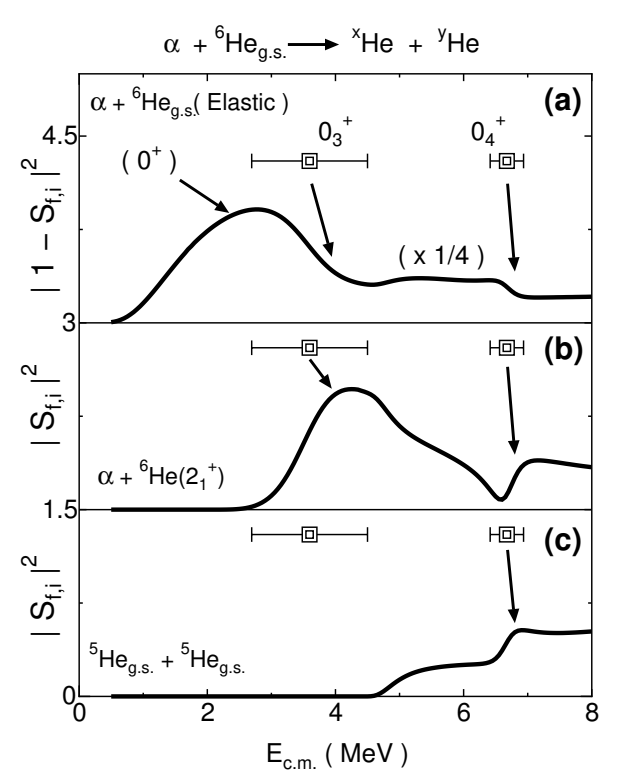

Figure 5. $\mathrm{S}$ matrices for the central collision of $\alpha+{ }^{6} \mathrm{He}_{\text {g.s. }}\left(\mathrm{J}^{\pi}=0^{+}\right)$. Panel (a) shows the $\mathrm{S}$ matrix of the elastic scattering, while (b) and (c) show those for the final channels of $\alpha+{ }^{6} \mathrm{He}\left(2{ }_{1}^{+}\right)$and ${ }^{5} \mathrm{He}_{\text {g.s. }}+{ }^{5} \mathrm{He}_{\text {g.s. }}$ (channel spin $I=0)$, respectively. The double squares represent the resonance energy obtained by the absorbing boundary condition, while the error bars, attached to the double squares, show the decay width of the resonance. The $\mathrm{S}$ matrices of the elastic scattering are divided by a factor of four. This figure is taken from Ref. [7] condition $(\mathrm{ABC})$ in order to identify the resonance energy and width more clearly $[18,19]$. In the ABC method, a negative imaginary potential is placed outside of the interaction region of the total system, and the continuum component contained in the wave function is absorbed. Due to this absorption, the resonant states are identified in the complex energy plane, and the resonance energy and the decay width can be calculated. The basic formulation of the ABC method is given in Refs. [18, 19]. The identified resonant levels are shown by the double squares with the error bars in all the panels of Fig. 5. The lower resonance at $E_{c . m} \sim 3.8 \mathrm{MeV}$ and higher resonance to $E_{c . m}$. $\sim 6.8 \mathrm{MeV}$ correspond to the $0_{3}^{+}$and $0_{4}^{+}$states, obtained in the structure 
analysis shown in the left panel of Fig. 1 . The $0_{3}^{+}$state, having a dominant component of $\alpha+{ }^{6} \mathrm{He}\left(2_{1}^{+}\right)$ $L=2$, corresponds to the extended VB state in Fig. 1, which is realized by the higher oscillation of the $\alpha-\alpha$ relative wave function. The $0_{4}^{+}$state is the compact MO states in Fig. 1, having the dominant configuration of $\left(\pi_{1 / 2}^{-}\right)^{2}$.

In the $\mathrm{S}$ matrix for the inelastic scattering of $\alpha+{ }^{6} \mathrm{He}_{\text {g.s. }} \rightarrow \alpha+{ }^{6} \mathrm{He}\left(2_{1}^{+}\right)$(Panel (b) of Fig. 5), there is a strong peak at $E_{c . m}$. $\sim 4 \mathrm{MeV}$, which nicely corresponds to the $0_{3}^{+}$state in resonance energy and width. As indicated by the arrow, the enhancement of the $S$ matrix at $E_{c . m} \sim 4 \mathrm{MeV}$ is generated by the existence of the resonant $\mathrm{O}_{3}^{+}$state, which are identified under the absorbing boundary condition. Since the $0_{3}^{+}$state has a well developed $\alpha+{ }^{6} \mathrm{He}\left(2_{1}^{+}\right)$structure, this state is strongly excited in the final channel of the inelastic scattering. On the contrary, in one neutron transfer channel of ${ }^{5} \mathrm{He}_{\text {g.s. }}+{ }^{5} \mathrm{He}_{\text {g.s. }}$, shown in the panel (c), we can also confirm a small peak at $E_{c . m}$. $7 \mathrm{MeV}$ near the resonance of the $0_{4}^{+}$ state. As shown by the arrow, this small peak is generated by the resonance of the $0_{4}^{+}$state, which has the MO configuration of $\left(\pi_{1 / 2}^{-}\right)^{2}$ with a spatially compact structure. Because of a large difference in the structure between $\alpha+{ }^{6} \mathrm{He}_{\text {g.s. }}$ and $\left(\pi_{1 / 2}^{-}\right)^{2}$, it is difficult to excite the $0_{4}^{+}$resonance from the incident channel of $\alpha+{ }^{6} \mathrm{He}_{\text {g.s. }}$. This is the reason why the $\mathrm{S}$ matrix is not so strongly enhanced at the resonance energy of the $0_{4}^{+}$state. In addition to these two peaks, a broad structure exists at $E_{c . m} \sim 3 \mathrm{MeV}$ in the elastic channel (Panel (a)). This broad peak is not a resonance but a continuum-like state because we cannot find any respective resonance in the calculation of the absorbing boundary condition.

The reaction mechanism of the resonance formation in the $S$ matrices shown in Fig. 5 is basically same as that discussed in ${ }^{12} \mathrm{Be}$; specifically, the resonant structures are generated by the coupling of the intrinsic resonant states shown by the double circles and the scattering of the open channels, such as $\alpha+{ }^{6} \mathrm{He}_{\text {g.s. }}, \alpha+{ }^{6} \mathrm{He}\left(2_{1}^{+}\right)$, and ${ }^{5} \mathrm{He}_{\text {g.s. }}+{ }^{5} \mathrm{He}_{\text {g.s. }}$. The restricted calculation, which contains only these three open channels, cannot generate the sharp resonant structure in the scattering probability shown in Fig. 5.

The calculation of the $\mathrm{S}$ matrix is consistent to the recent observations. The broad $0^{+}$continuum in the calculation correspond to the broad $0^{+}$strength observed by Rogachev et al. [16], while a resonance-like $0^{+}$state is observed in the $\alpha$ scattering at the energy position close to the theoretical $0_{3}^{+}$resonance [20]. In Ref. [20], the low-energy scattering of $\alpha+{ }^{6} \mathrm{He}_{\text {g.s. }} \rightarrow \alpha+{ }^{6} \mathrm{He}\left(2_{1}^{+}\right)$has been performed, and the respective angular distributions has been analyzed. A candidate of $L=2$ resonance is suggested at the energy of $E_{c . m .}=4.4 \mathrm{MeV}\left(E_{x} \sim 11.8 \mathrm{MeV}\right)$, which is almost the same as the resonance energy of the theoretical $0_{3}^{+}$state with the $\alpha+{ }^{6} \mathrm{He}\left(2_{1}^{+}\right) L=2$ structure. In a deep comparison of the theoretical calculation with the experiments, more precise experimental data and the extended calculation on the differential cross section are indispensable.

\subsection{Coulomb shift in ${ }^{10} \mathrm{Be}$ and ${ }^{10} \mathrm{C}$}

We apply the GTCM to ${ }^{10} \mathrm{C}=\alpha+\alpha+P+P$, which is the mirror system of ${ }^{10} \mathrm{Be}$, and the Coulomb shift is investigated. The GTCM calculation for ${ }^{10} \mathrm{C}$ is performed in the similar manner by adding the Coulomb interaction of $\alpha-P$. We calculate the bound and continuum spectra under the bound and scattering boundary conditions, respectively. Figure 6 shows the energy levels of ${ }^{10} \mathrm{Be}$ (left levels) and ${ }^{10} \mathrm{C}$ (right levels). In the individual spectra, the lowest four $0^{+}$levels are plotted except for the broad continuum bump at $E_{c . m}$. $\sim 3 \mathrm{MeV}$ shown in Figs. 1 and 5. As we have already confirmed in Fig. 1, the internal structures of the $0_{1}^{+}$and $0_{2}^{+}$states are the MO configurations of $\left(\pi_{3 / 2}^{-}\right)^{2}$ and $\left(\sigma_{1 / 2}^{+}\right)^{2}$, respectively, in which two valence nucleons perform the single particle motion around two $\alpha$ cores. On the contrary, the unbound $0_{3}^{+}$and $0_{4}^{+}$states have the $\alpha+{ }^{6} \mathrm{He}\left(2^{+}\right)$and $\left(\pi_{1 / 2}^{-}\right)^{2}$, respectively.

In the ${ }^{10} \mathrm{C}$ spectra, all of the energy levels shift to the higher energy region because of the Coulomb repulsion of the $\alpha-P$ system. The magnitudes of the shifts are not common among the levels but they 
depend on the energy levels. The shift of the $0_{1}^{+}$state is $2.3 \mathrm{MeV}$, which is the largest of all the energy levels. This prominent shift is due to the spatially compact structure of the $\left(\pi_{3 / 2}^{-}\right)^{2}$ configuration. The $\pi_{3 / 2}^{-}$orbit, which has the dominant component of the $0 p_{3 / 2}$ orbit, strongly reduce the $\alpha-\alpha$ distance, and the $0_{1}^{+}$state has a spatially compact structure similar to the shell model-like structure. Therefore, the effect of the Coulomb repulsion is prominent for the compact $0_{1}^{+}$state if the valence neutrons are replaced by the protons.

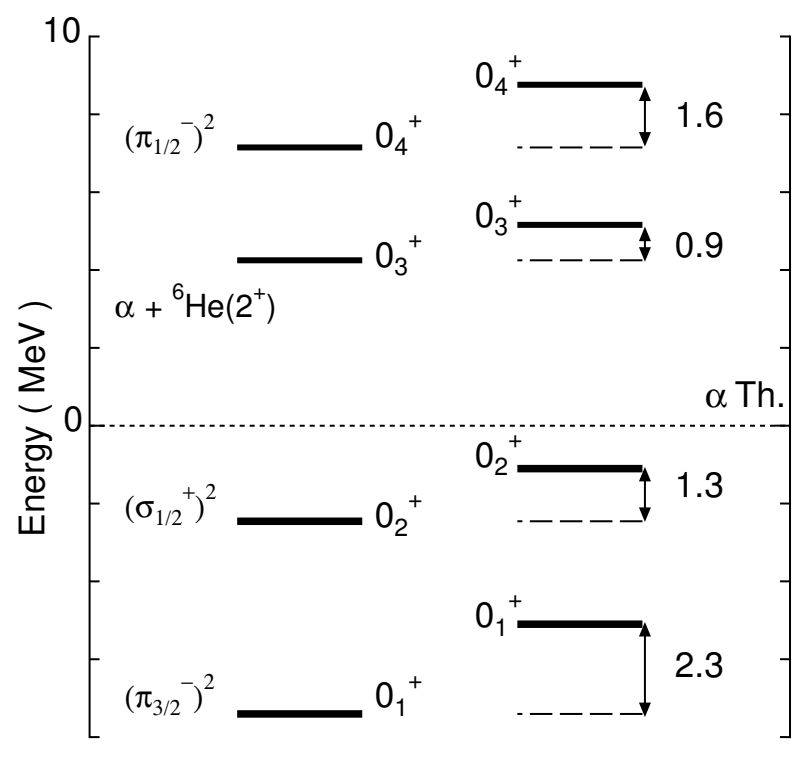

Figure 6. Energy levels of the ${ }^{10} \mathrm{Be}$ (left levels) and ${ }^{10} \mathrm{C}$ (right levels) nuclei. The $\alpha$ threshold is set to be zero energy. At the ${ }^{10} \mathrm{C}$ spectra, the corresponding levels to ${ }^{10} \mathrm{Be}$ are plotted by the dashed line. The numbers attached to the levels show the Coulomb shifts. The shifts are shown in units of $\mathrm{MeV}$.

On the contrary, the Coulomb shift of the $0_{2}^{+}$state is $1.3 \mathrm{MeV}$, which is smaller than the shift of the ground $0_{1}^{+}$state, $2.3 \mathrm{MeV}$. The reduction of the shift for $0_{2}^{+}$is due to the spatially extended structure of the $\sigma_{1 / 2}^{+}$orbit, which has a large mixture of the $\mathrm{S}$-wave component. The large mixing of the S-wave component in the valence orbit leads to the enhancement of the $\alpha-\alpha$ clustering. Since the repulsive effect of the Coulomb interaction is suppressed in the spatially extended configuration, the Coulomb shift becomes small in the $0_{2}^{+}$state. Due to the difference of the Coulomb repulsion, the excitation energy of the $0_{2}^{+}$in ${ }^{10} \mathrm{C}$ is smaller by about $1 \mathrm{MeV}$ than that of $0_{2}^{+}$in ${ }^{10} \mathrm{Be}$. This shift of the excitation energy is consistent to the recent compilation of the energy levels of the ${ }^{10} \mathrm{C}$ nucleus [21].

In the continuum energy region above the $\alpha$ threshold, the difference of the Coulomb shift is also seen in the $0_{3}^{+}$and $0_{4}^{+}$states; $0.9 \mathrm{MeV}$ for the former state and $1.6 \mathrm{MeV}$ for the latter state. Since the difference of the Coulomb barrier is about $1.5 \mathrm{MeV}$ between $\alpha+{ }^{6} \mathrm{He}$ and $\alpha+{ }^{6} \mathrm{Be}$, the Coulomb shift of the $0_{3}^{+}$state is suppressed in comparison to the shift of the Coulomb barrier. The $0_{3}^{+}$state has a dominant component of resonant $\alpha+{ }^{6} \mathrm{He}\left(2^{+}\right)$state with the $\mathrm{D}$-wave relative motion, but there is a considerable mixture of $\alpha+{ }^{6} \mathrm{He}_{\text {g.s. }}$. with the $\mathrm{S}$-wave component. This $\mathrm{S}$-wave mixture is due to the existence the broad $\alpha+{ }^{6} \mathrm{He}_{\text {g.s. }}$ strength just below the $0_{3}^{+}$state, as shown in Fig. 5. Therefore, the $0_{3}^{+}$state has the extended and dilute binary structure, which leads to the suppression of the Coulomb shift. On the contrary, the $0_{4}^{+}$state has the dominant component of the compact MO configuration of 
$\left(\pi_{1 / 2}^{-}\right)^{2}$, which has a large amplitude of the wave function inside of the interaction region. Thus, the Coulomb shift is enhanced in the compact $0_{4}^{+}$state.

In all the spectra, the Coulomb shift is reduced for the spatially extended state $\left(0_{2}^{+}\right.$and $\left.0_{3}^{+}\right)$, which has a large mixture of the $\mathrm{S}$-wave component, while the shift is enhanced for the compact non-S-wave states $\left(0_{1}^{+}\right.$and $\left.0_{4}^{+}\right)$. Therefore, these suppressed Coulomb shift confirmed in the present calculation are basically same as the Thomas-Ehrman Shift (TES), which was originally discussed in the ${ }^{17} \mathrm{O}$ and ${ }^{17} \mathrm{~F}$ systems [9]. The Coulomb suppression in ${ }^{10} \mathrm{Be}$ and ${ }^{10} \mathrm{C}$, which are shown in Fig. 6, correspond to the extension of the TES to the cluster degrees of freedom of $\alpha+\alpha+2 N$ and hence, the present shift should be called the "Cluster Thomas-Ehrman shift (Cluster TES)". The shift of the excitation energy in the Cluster TES is about $1 \mathrm{MeV}$ for the $0_{2}^{+}$state. This shift is much larger than the shift discussed in the traditional TES, which is about $0.4 \mathrm{MeV}$ for the $1 / 2^{+}$state with respect to the ground $5 / 2^{+}$state.

Although we have discussed the energy levels in the mirror systems of ${ }^{10} \mathrm{Be}$ and ${ }^{10} \mathrm{C}$, the present calculation also predicts the asymmetry in the monopole transition from $0_{1}^{+}$to $0_{2}^{+}$; specifically, the $0_{1}^{+}$ $\rightarrow 0_{2}^{+}$strength in ${ }^{10} \mathrm{C}$ is about twice as large as the respective strength in ${ }^{10} \mathrm{Be}$. Due to the suppression of the Coulomb shift for the $0_{2}^{+}$state, the excitation energy of $0_{2}^{+}$in ${ }^{10} \mathrm{C}$ is reduced in comparison to the respective excitation energy in ${ }^{10} \mathrm{Be}$. This reduction ${ }^{10} \mathrm{C}$. A detailed analysis of the monopole transitions will be shown in forthcoming papers.

\section{Summary and discussion}

We have formulated the generalized two-center cluster model (GTCM), which can handle the formation of the compact MO structure and the extended VB structure in a consistent manner [7, 8]. The basic structure of these two configurations, MOs and VBs, is almost same as the electrons' bonds employed in quantum chemistry, but GTCM can describe their dynamical transition induced by the Hamiltonian of the total many-body systems; specifically, this model can generate the compact MO structure at a small $\alpha-\alpha$ distance, while, in the asymptotic region, the extended VB structure are naturally formed.

First, GTCM has been applied to the structure problem in ${ }^{10,12} \mathrm{Be}$, and the energy spectra have been calculated under the appropriate boundary conditions. In ${ }^{10,12} \mathrm{Be}$, there is one to one correspondence between the energy levels and the respective threshold. This correspondence means that the excited levels appear according to the threshold rule. The structure of the neutrons' orbit prominently changes from level to level as a function of the excitation energy. In the bound region, the covalent MO structures are dominant, and the ionic VB structures mainly appears in the unbound region. The bound and resonant levels are compared with all the recent observations. The results of the GTCM calculation is completely consistent to the level scheme observed over a wide energy region, which contains the low-lying intruder $0_{2}^{+}$below the $\alpha$ decay threshold and the continuum states above the $\alpha$ threshold [4-6]. In particular, the results of ${ }^{12} \mathrm{Be}$ is nicely reproduce the degenerate feature of the levels in the unbound retion [5].

Secondly, GTCM is applied to the resonant scattering of ${ }^{8,6} \mathrm{He}$ by an $\alpha$ target. In $\alpha+{ }^{8} \mathrm{He}_{\text {g.s. }}$, we have formulated the closed state method to handle the scattering problem in a close relationship with the structure information on the compound system of ${ }^{12} \mathrm{Be}$. In the closed state method, the coupling of the closed state, which are obtained from the bound-state-like boundary condition, and the open channels is explicitly solved. The formation of the sharp resonance can be naturally described by the mixing of the open channels and the closed states, which contains all the structure correlations inside of the interaction region. We have focused on two neutron transfer, $\alpha+{ }^{8} \mathrm{He}_{\text {g.s. }} \rightarrow{ }^{6} \mathrm{He}_{\text {g.s. }}+{ }^{6} \mathrm{He}_{\text {g.s. }}$, and pointed out a characteristic decay scheme in the central $J^{\pi}=0^{+}$collision. Similar reaction analysis has been performed for the $\alpha+{ }^{6} \mathrm{He}_{\text {g.s. }}$, and we have predicted a prominent resonance in the 
inelastic scattering of $\alpha+{ }^{6} \mathrm{He}_{\text {g.s. }} \rightarrow \alpha+{ }^{6} \mathrm{He}\left(2^{+}\right)$. In both of the systems, the obtained reaction scheme is consistent to the recent observations on the unbound states above the $\alpha$ threshold $[5,6,16,17,20]$.

Finally, we have applied GTCM to ${ }^{10} \mathrm{C}=\alpha+\alpha+2 P$, which is the mirror partner of ${ }^{10} \mathrm{Be}=\alpha+\alpha$ $+2 N$, and the Coulomb shift in the lowest four $0^{+}$states is analyzed. The present calculation predicts the suppression of the Coulomb shift for the spatially extended states of $0_{2,3}^{+}$, which are induced by the development of the $\alpha$ clustering. Since the spatially-extended cluster structure involves the large mixture of the S-wave component, the Coulomb suppression is attributed to the anomalous feature of the S-wave orbit. The suppressed Coulomb shift presented here is basically same as the ThomasEhrman Shift (TES), which is discussed in the S-wave orbit for the single nucleon degree of freedom [9]. Therefore, we call the shift of the excitation energy of $0_{2}^{+}$found in ${ }^{10} \mathrm{Be}$ and ${ }^{10} \mathrm{C}$ "Cluster TES".

In stable $N=Z$ systems, the energy positions and their decay width into the $\alpha$ particle were the signatures in identifying an excited $0^{+}$level as an $\alpha$ cluster state. Although the Coulomb shift is meaningless in the $N=Z$ system, the Coulomb shift originated from the Cluster TES will be a new sign to identify the cluster structure in $N \neq Z$ systems. In the analysis of the Coulomb shift, the unified treatment of the structure and reaction is very useful because a large part of the excited energy levels in $N \neq Z$ sytems appears as the resonant state above the particle decay threshold. The systematic studies of various mirror systems are now under progress.

\section{References}

[1] H. Furutani et al., Suppl. Prog. Theor. Phys. 68, 193 (1980); M. Seya, M. Kohn, and S. Nagata, Prog. Theor. Phys. 65, 204 (1981).

[2] N. Itagaki and S. Okabe, Phys. Rev. C 61, 044306 (2000).

[3] N. Itagaki, S. Okabe and K. Ikeda, Phys. Rev. C 62, 034301 (2000), and references therein.

[4] M. Freer et al., Phys. Rev. Lett.82, 1383 (1999) ; Phys. Rev. C63, 034301 (2001).

[5] A. Saito et al., Mod. Phys. Lett. A 251858 (2010), and references therein.

[6] Z. H. Yang, et al., Phys. Rev. Lett. 112, 162501 (2014), and references therein.

[7] M. Ito, Phys. Rev. C 85, 044308 (2012); M. Ito and K. Ikeda, Rep. Prog. Phys. 77, 096301 (2014), and references therein.

[8] M. and N. Itagaki, Phys. Rev. C 78 011602(R) (2008); M. Ito and D. Suzuki, Phy. Rev. C 84 014608 (2011), and references therein.

[9] Bohr A and Mottelson B R 1969 Nuclear Structure vol I (New York: Benjamin) p 320.

[10] H. Horiuchi, Suppl. Prog. Theor. Phys. 62, 90 (1977).

[11] M. Ito, K. Yabana, T. Nakatsukasa and M. Ueda, Phys. Lett. B 637, 53 (2006).

[12] A. B. Volkov, Nucl. Phys. 74, 33 (1965).

[13] N. Yamaguchi, T. Kasahara, S. Nagata, and Y. Akaishi, Prog. Theor. Phys. 62, 1018 (1979).

[14] M. Ito, Y. Hirabayashi, and Y. Sakuragi, Eur. Phys. Jour. 5, 373 (1999).

[15] K. Ikeda, H. Horiuchi, and S. Saito, Prog. Theor. Phys. 68, 1 (1980), and references therein.

[16] G. V. Rogachev et al., Prog. Theor. Phys. Suppl. 196, 184,(2012), and references therein.

[17] M. Freer, Private Communication (2014).

[18] Y. Takenaka, et al., Prog. Exp. Theor. Phys. 2014, 113D04 (2014), and references therein.

[19] M. Iwasaki, R. Otani, Y. Takenaka, and M. Ito, Prog. Exp. Theor. Phys. 2015 023D01, (2015).

[20] D. Suzuki et al., Phys. Rev. C 87, 054301 (2013).

[21] V. Z. Goldberg and G. V. Rogachev, Phys. Rev. C86, 044314 (2012). 